\title{
Optimization of mediastinal staging in potential candidates for stereotactic radiosurgery of the chest
}

\author{
Devadatta Sarwate, MD, ${ }^{\text {a }}$ Saiyad Sarkar, MD, ${ }^{\text {a,b }}$ William S. Krimsky, MD, ${ }^{\text {a,b }}$ Constantine M. Burgan, MD, ${ }^{\text {a }}$ \\ Kalpesh Patel, MD, ${ }^{\mathrm{a}}$ Ruth Evans, RN, CNOR, OCN, ${ }^{\mathrm{a}}$ and Daniel P. Harley, MD ${ }^{\mathrm{b}}$
}

Objective: Patients with medically inoperable nonsmall-cell lung cancer generally have limited staging of the mediastinum using computed tomography and combined positron emission tomography and computed tomography, before stereotactic radiosurgery. Historical data have demonstrated the superiority of tissue sampling techniques such as endobronchial ultrasonography and mediastinoscopy compared with imaging studies in accurately determining the nodal stage. We believe, that at a minimum, mediastinal interrogation with endobronchial ultrasonography should be performed before patients undergo stereotactic radiosurgery.

Methods: A retrospective review of 59 consecutive patients undergoing bronchoscopic fiducial marker placement as potential candidates for stereotactic radiosurgery was done. All these patients had undergone endobronchial ultrasonography to assess the mediastinum. Transbronchial needle aspirates were taken using standard criteria defined by a lymph node size greater than $5 \mathrm{~mm}$ in diameter and/or in the appropriate lymph node drainage pathway. The biopsies were reviewed by the institution's pathologists.

Results: Mediastinal lymph node specimens were not taken in 9 patients because they did not meet our criteria. Of the 50 patients who underwent mediastinal lymph node sampling, 10 had evidence of nodal involvement. On review, 2 of these 10 patients had evidence of mediastinal adenopathy on computed tomography. After excluding those 2 patients, the mediastinal lymph nodes were positive for metastatic disease in $8(16 \%)$ of 50 patients without previous radiographic evidence of disease. These patients were previously thought to be suitable candidates for stereotactic radiosurgery. Also 5 of 10 patients with endobronchial ultrasound-positive lymph nodes had had positron emission tomography-negative findings in the mediastinum. Finally, $10 \%$ of the patients suspected to have stage II or III were downstaged with endobronchial ultrasonography and considered for stereotactic radiosurgery.

Conclusions: Endobronchial ultrasonography-transbronchial needle aspirates is more accurate than computed tomography and positron emission tomography in staging the mediastinum, can be performed with minimal morbidity, and should be considered for all patients considered candidates for stereotactic radiosurgery. (J Thorac Cardiovasc Surg 2012;144:81-6)

Effective treatment of lung cancer necessitates accurate diagnosis and staging. There is little disagreement that for patients considered for surgical resection of nonsmall-cell lung cancer (NSCLC), staging, in most cases, necessitates investigations other than computed tomography (CT) and positron emission tomography (PET). ${ }^{1}$ A large cohort of clinical stage IA patients with NSCLC exists who would be unable to tolerate the rigors of a surgical resection. In these patients, the use of stereotactic body radiotherapy

From the Departments of Pulmonary Medicine ${ }^{\mathrm{a}}$ and Thoracic Surgery, ${ }^{\mathrm{b}}$ Franklin Square Hospital, Baltimore, Md

Disclosures: Authors have nothing to disclose with regard to commercial support.

Read at the 91st Annual Meeting of The American Association for Thoracic

Surgery, Philadelphia, Pennsylvania, May 7-11, 2011.

Received for publication May 10, 2011; revisions received Feb 23, 2012; accepted for publication March 12, 2012; available ahead of print April 12, 2012.

Address for reprints: Devadatta Sarwate, MD, Department of Pulmonary Medicine, Franklin Square Hospital, 9000 Franklin Square Drive, Baltimore, MD 21237

(E-mail: devadatta.sarwate@gmail.com).

$0022-5223 / \$ 36.00$

Copyright (C) 2012 by The American Association for Thoracic Surgery

doi:10.1016/j.jtcvs.2012.03.004
(SBRT) systems can offer significant improvements in the treatment and local control of stage IA and IB NSCLCs. ${ }^{2-4}$

At Franklin Square Hospital Center in Baltimore, Md, Cyberknife with Synchrony (Accuray Robotic Radiosurgery Systems; Accuray, Sunnyvale, Calif) is used to treat medically inoperable stage IA and B NSCLC. This system achieves significant improvements in local control rates in patients with stage I NSCLC who were not candidates for surgical resection. ${ }^{5,6}$ The use of SBRT in patients with stage II, III, and IV NSCLC will be ineffective and potentially harmful. For most lung tumors, the tracking system of Cyberknife with Synchrony requires placement of fiducial markers (FMs) placed in or near the tumor. We have previously published our data regarding the efficacy of FM placement using a combination of electromagnetic navigational bronchoscopy and endobronchial ultrasonography (EBUS). ${ }^{7}$ It is critical to recognize that this method has the advantage of performing EBUS interrogation of the mediastinum at FM placement, allowing for the detection of any unsuspected synchronous mediastinal and/or hilar nodal disease spread. This results in more accurate staging 


$$
\begin{aligned}
& \text { Abbreviations and Acronyms } \\
& \begin{array}{ll}
\text { CT } & =\text { computed tomography } \\
\text { FM } & =\text { fiducial marker } \\
\text { PET } & =\text { positron emission tomography } \\
\text { EBUS } & =\text { endobronchial ultrasonography } \\
\text { NSCLC } & =\text { nonsmall-cell lung cancer } \\
\text { SBRT } & =\text { stereotactic body radiotherapy } \\
\text { SRS } & \text { stereotactic radiosurgery } \\
\text { TBNA } & =\text { transbronchial needle aspiration }
\end{array}
\end{aligned}
$$

but potentially alters the treatment approach in patients with existing nodal disease and in patients with falsepositive lymph node involvement on CT and PET scans.

The present study evaluated our experience using the deployment of ultrasound interrogation of the mediastinum in patients with clinical stage IA, IB, and II NSCLC at FM placement to achieve accurate disease staging and to ensure optimal treatment.

\section{METHODS}

From June 2009 to July 2010, 59 consecutive patients (Table 1) who were considered medically inoperable and had histologically proven clinical stage I NSCLC were evaluated for treatment by a multidisciplinary lung cancer tumor board at the Franklin Square Hospital Center. The tumor board members included physicians from the departments of thoracic surgery, pulmonary medicine, thoracic oncology, and pathology, and treatment recommendations were achieved by a majority consensus. After review, the patients who were potential candidates for SBRT according to the CT and PET staging and poor physiologic status were referred for FM placement. The age range of these patients was 52 to 91 years (mean age, 72.2 years). Of the 59 patients, 33 were men and 26 were women. The institutional review board of MedStar Health, the parent corporation of Franklin Square Hospital Center, approved the data collection and analysis of these patients and waived patient consent for the present review.

All patients had undergone noncontrast-enhanced CT scans of the chest configured with 1-mm by 1-mm slices in the Digital Imaging and Communications in Medicine format. All patients had undergone whole body PET scans. All the studies were reviewed by an independent radiologist for the present study and by an independent radiologist for the lung cancer tumor board. Patients with unequivocal evidence of metastatic disease were not considered for SBRT to the lung. The PET scans were all PET-CT scans and were all performed at 1 imaging facility. The CT scans were all performed at the Franklin Square Hospital Center.

All procedures were performed separately by 2 interventional pulmonologists with extensive experience in both EBUS and electromagnetic navigational bronchoscopy. The procedures were performed with the patient under a light general anesthetic administered orally and chosen by the anesthesiologist. All patients were positioned on an electromagnetic location board at the beginning of each procedure. EBUS was performed using an adult 3.0-mm working channel adult bronchoscope with an EBUS probe. Our technique for using electromagnetic navigational bronchoscopy, EBUS, and FM placement and confirmation has been previously described. ${ }^{7}$ At FM placement, EBUS was used to interrogate the mediastinum, and the International Association for the Study of Lung Cancer lymph node stations were scanned distally to proximally, starting in the hilum and moving proximally, followed by interrogation of the subcarinal, pre- and postcarinal nodes, and paratracheal nodes.
TABLE 1. Patient characteristics

\begin{tabular}{lc}
\hline \multicolumn{1}{c}{ Characteristic } & Value \\
\hline Age (y) & \\
Mean & 72.2 \\
Range & $52-91$ \\
Gender (n) & \\
Male & 33 \\
Female & 26 \\
Additional comorbidities (n) & \\
Cardiovascular & 24 \\
Other & 7 \\
Lung involved (n) & \\
Right & 35 \\
Left & 24 \\
Tumor location (n) & \\
Central & 12 \\
Peripheral & 47 \\
\hline
\end{tabular}

We used generally accepted criteria for lymph node sampling. ${ }^{8}$ The lymph nodes with a short axis diameter greater than $5 \mathrm{~mm}$ were identified and sampled, providing they were in the appropriate distribution for drainage from the suspect lesion. A 21- or 22-gauge transbronchial needle aspiration (TBNA) needle was inserted into each of these areas and a specimen obtained. Separate needles were used for each station to avoid the potential of cross-contamination. A cytopathologist was available to assess the adequacy of the aspirated material at surgery, but the final diagnosis was deferred until the material had been reviewed by 2 separate histopathologists. Smaller lymph nodes suspicious for metastatic spread on EBUS were also aspirated. This was performed at the discretion of the endoscopist. Negative nodes were defined by the presence of benign anthracotic tissue and negative flow cytometry analysis findings.

\section{RESULTS}

All patients in the present study were classified inoperable. In most cases, this was because of advanced pulmonary disease. This was defined by the pulmonary function test results. Those patients with a forced expiratory volume in 1 second of less than $40 \%$ of predicted and a diffusion capacity of the lung of less than $40 \%$ of predicted were considered to have severe obstructive lung disease. ${ }^{9}$ In addition to their existing pulmonary disease, 24 patients $(40 \%)$ had cardiovascular disease and 7 had other comorbidities; most were stroke and/or other malignancies. Of the 59 patients, $16(27 \%)$ were octogenarians with poor functional reserve due to age-related disability. NSCLC was diagnosed in 58 patients, and 1 patient, suspected to have early-stage NSCLC and considered a potential candidate for SBRT, was diagnosed with small cell lung cancer from an EBUS-directed biopsy. Twelve patients had local recurrence after previous surgical resection. One patient had undergone a previous pneumonectomy. Of the 59 tumors, 35 were located on the right and 24 on the left; 12 tumors were central in location and 47 were peripheral. The mean tumor size was $2.7 \mathrm{~cm}$. All patients underwent disease staging using the 7 th edition TNM staging system. 


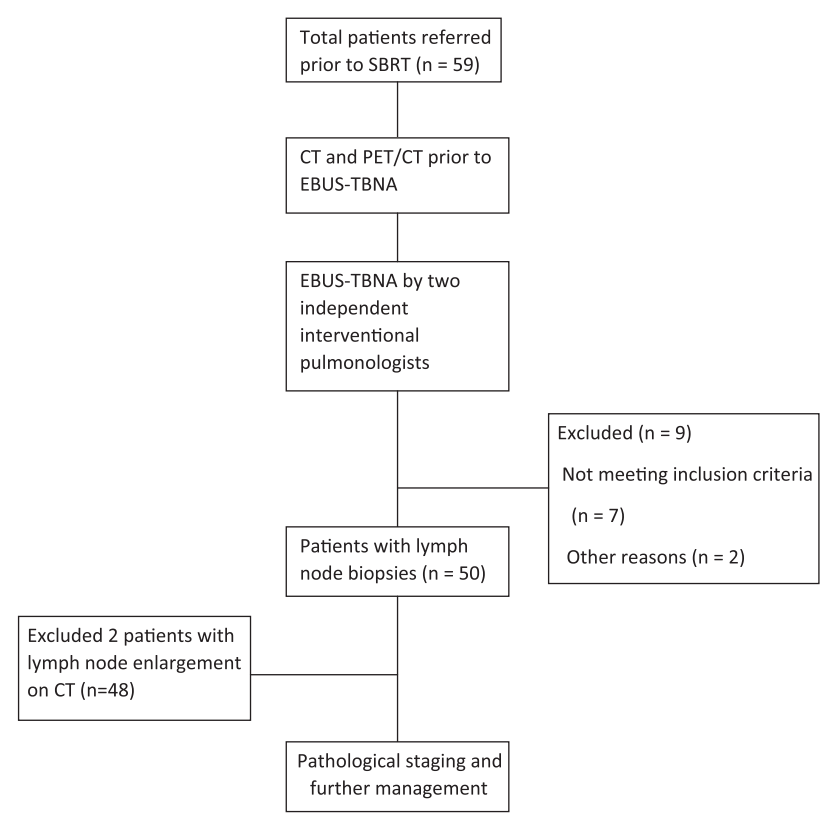

FIGURE 1. Flow of participants through each stage of selection process. $S B R T$, Stereotactic body radiotherapy; $C T$, computed tomography; PET, positron emission tomography; EBUS, endobronchial ultrasonography; $T B N A$, transbronchial needle aspiration.

EBUS was performed at FM placement in all patients. Lymph node biopsies were not taken in 9 patients, 7 of whom had failed to meet the criteria identified previously for EBUS sampling. Of the remaining 2 patients, 1 did not undergo lymph node sampling because of tortuous airways and 1 because of the presence of an endoluminal tumor (Figure 1).

A total of 92 lymph nodes were biopsied, 59 in the mediastinum and 33 in the hila (Tables 2-4). Of the 50 patients who underwent EBUS-directed biopsies, $10(20 \%)$ had

TABLE 2. EBUS-TBNA positive pathologic findings compared with CT and PET findings in mediastinum

\begin{tabular}{llllc}
\hline Pt. no. & CT report & PET report & Tumor location & $\begin{array}{c}\text { Stations positive } \\
\text { on pathologic } \\
\text { examination }\end{array}$ \\
\hline 1 & Negative & Negative & Peripheral & $7,10 \mathrm{~L}$ \\
2 & Negative & Negative & Central & $4 \mathrm{R}, 7$ \\
3 & Negative & Negative & Peripheral & $4 \mathrm{R}, 10 \mathrm{R}$ \\
4 & Negative & Positive & Central & $7,10 \mathrm{~L}$ \\
5 & Negative & Positive & Peripheral & 7 \\
6 & Negative & Positive & Peripheral & 7 \\
7 & Negative & Positive & Peripheral & $4 \mathrm{R}$ \\
8 & Negative & Negative & Peripheral & 7 \\
9 & Positive & Negative & Peripheral & 7 \\
10 & Positive & Positive & Peripheral & $7,12 \mathrm{R}$ \\
\hline
\end{tabular}

$E B U S$, Endobronchial ultrasonongraphy; $T B N A$, transbronchial needle aspiration; $C T$, computed tomography; $P E T$, positron emission tomography; Pt. no., patient number; $L$, left; $R$, right.
TABLE 3. Lymph node stations by EBUS-TBNA with result

\begin{tabular}{lcc}
\hline \multicolumn{1}{c}{ Lymph node station } & Positive & Negative \\
\hline $2 \mathrm{~L}$ & 0 & 3 \\
$4 \mathrm{R}$ & 3 & 7 \\
$4 \mathrm{~L}$ & 0 & 6 \\
7 & 8 & 32 \\
$10 \mathrm{~L}$ & 3 & 6 \\
$10 \mathrm{R}$ & 1 & 11 \\
$11 \mathrm{~L}$ & 0 & 3 \\
$11 \mathrm{R}$ & 0 & 4 \\
$12 \mathrm{~L}$ & 0 & 1 \\
$12 \mathrm{R}$ & 1 & 3 \\
Regional lymph node involvement & & \\
Mediastinal (N2 nodes) & 11 & 48 \\
Hilar (N1 nodes) & 5 & 28 \\
\hline
\end{tabular}

EBUS, Endobronchial ultrasonography; TBNA, transbronchial needle aspiration; $L$, left; $R$, right.

evidence of mediastinal lymph node involvement according to the pathology results. On further review of the CT results, 2 of those 10 patients were believed to have equivocal findings on the CT scan suggestive of mediastinal lymph node enlargement. After excluding those patients from the analysis, the percentage of unexpected positive results decreased to $16.6 \%$ (8/48 patients). Additionally, occult (PET/CT-negative) disease was present in roughly $10 \%$ of these patients (5/48). Also, 4 patients with PET-positive scans, who would have been excluded from treatment on the basis of those results, were found to not have metastatic nodal spread, defined as the absence of malignant cells in the presence of small bland lymphocytes or fragments of benign lymphoid tissue on histopathologic examination.

All patients tolerated the procedure well. No anesthesiarelated complications occurred. Some patients had bloodstreaked sputum after EBUS-TBNA; however, no patients were readmitted to the hospital. No pneumothoraces developed, and all patients were discharged in stable condition.

\section{DISCUSSION}

After the tissue diagnosis of inoperable NSCLC has been established or with patients in whom the clinical suspicion is high and stereotactic radiosurgery (SRS) is recommended, consideration must turn toward the determination of the disease extent, or stage, because this has a direct effect on the management and prognosis. From the available

TABLE 4. Comparison of imaging results with EBUS pathologic findings in mediastinum $(n=50)$

\begin{tabular}{lcc}
\hline Variable & EBUS positive & EBUS negative \\
\hline CT positive & 2 & 9 \\
CT negative & 8 & 31 \\
PET positive & 5 & 4 \\
PET negative & 5 & 36 \\
\hline
\end{tabular}

EBUS, Endobronchial ultrasonography; $C T$, computed tomography; PET, positron emission tomography. 
published data, ${ }^{10-13}$ these patients typically undergo limited staging of the mediastinum by CT and PET.

CT scanning of the chest has a sensitivity and specificity of $51 \%$ and $86 \%$ for identifying mediastinal lymph nodes metastasis, respectively, when the nodes are greater than $1 \mathrm{~cm}$ in the short-axis diameter. In patients with clinical T1N0 tumors, nodal metastasis can be missed in $5 \%$ to $15 \%$ of cases using CT evaluation of the mediastinum. ${ }^{14}$ Given the limitations of its sensitivity and specificity, it would be unfortunate to rely solely on CT scanning for the evaluation of mediastinal lymph nodes in the patient selection for SRS.

PET is more accurate than CT in detecting mediastinal lymph node metastasis. The sensitivity and specificity has been found to be $74 \%$ and $85 \%$, respectively. However, this is also not ideal for several reasons. PET has limited anatomic and spatial resolution, with the lower limit of size approximately $7 \mathrm{~mm}$ to $10 \mathrm{~mm}$. Also, no standardized quantitative criteria are available for what constitutes an abnormal PET finding in the mediastinum. Specifically, it is difficult to differentiate between malignant and nonneoplastic conditions such as granulomatous, inflammatory, or infectious disease. Furthermore, false-positive PET findings can occur in $25 \%$ of patients with enlarged mediastinal nodes. This can lead to the denial of the optimal treatment strategy. In contrast, false-negative PET findings can lead to inappropriate treatment. In a meta-analysis by Gould and colleagues, ${ }^{15} 20 \%$ of patients with normal-size nodes but with malignant involvement had false-negative PET findings.

EBUS-TBNA has a sensitivity of $89 \%$, specificity of $100 \%$, and negative predictive value of $98.9 \%$ in patients with negative CT and PET findings for nodal metastasis. ${ }^{16}$ Compared with surgical staging in patients with clinical stage I tumors, it has also been found to have comparable results.

At our institution, we have adopted the clinical practice of investigating the mediastinum with EBUS at bronchoscopic FM placement in patients considered for SRS by our multidisciplinary tumor board. We believe that the mediastinum should be investigated using EBUS-TBNA. We do not routinely perform mediastinoscopy in this group of patients, because EBUS-TBNA is a less-invasive procedure and has the advantage of being able to assess more nodal stations than mediastinoscopy, including N1 nodes. We acknowledge that the current published false-negative rate for EBUS-TBNA is greater than that of mediastinoscopy, although, again, as noted, these 2 modalities have been found to have comparable results for stage I tumors.

Our results have confirmed the dissonance among CT, PET, and EBUS-TBNA in accurately staging the mediastinum. Its results are comparable to, and in the case of occult $\mathrm{N} 1$ disease superior to, those of mediastinoscopy. Of the patients in the present study who were scheduled for SRS,
$16 \%$ were upstaged by diagnosing occult mediastinal spread. These patients were re-evaluated for alternative treatment modalities because SRS alone would clearly have been suboptimal in these settings. Patients with positive PET findings should also always undergo invasive staging of the mediastinum to ensure they are not denied optimal treatment. This was highlighted in that just less than $50 \%$ (4/9) of patients with PET positive findings in the mediastinum were downstaged such that they became eligible for SRS. A bronchoscopic approach to staging the mediastinum also allows other procedures to be performed in the same session. A biopsy of lesions is 1 such advantage. In 1 of our patients, the lesion yielded small cell lung cancer, resulting in a change in management.

Our study also raises the question of the accuracy of previous SRS studies performed without any invasive mediastinal staging. EBUS-TBNA will increase the accuracy of future studies evaluating SRS, including both FM and FM-less systems.

\section{CONCLUSIONS}

The use of EBUS-TBNA should be considered for all patients being evaluated for SRS. It is highly accurate in all patients with and without mediastinal lymph node enlargement on CT and with or without PET activity in the mediastinum. It is an appropriate choice for the primary procedure, because it is well tolerated, carries minimal morbidity, and allows for additional pulmonary procedures in the same setting.

\section{References}

1. Detterbeck FC, Jantz MA, Wallace M, Vansteenkiste J, Silvestri GA, American College of Chest Physicians. Invasive mediastinal staging of lung cancer: ACCP evidence-based clinical practice guidelines (2nd edition). Chest. 2007;132(3 Suppl):202S-20S

2. Vahdat S, Oermann EK, Collins SP, Yu X, Abedalthagafi M, Debrito P, et al. CyberKnife radiosurgery for inoperable stage IA non-small cell lung cancer: $18 \mathrm{~F}$-fluorodeoxyglucose positron emission tomography/computed tomography serial tumor response assessment. J Hematol Oncol. 2010;3:6.

3. Brown WT, Wu X, Amendola B, Perman M, Han H, Fayad F, et al. Treatment of early non-small cell lung cancer, stage IA, by image-guided robotic stereotactic radioablation-CyberKnife. Cancer J. 2007;13:87-94.

4. Timmerman R, Paulus R, Galvin J, Michalski J, Straube W, Bradley J, et al. Stereotactic body radiation therapy for inoperable early stage lung cancer. JAMA. 2010;303:1070-6.

5. Whyte R, Crowner R, Murphy MJ, Martin DP, Rice TW, DeCamp MM, et al. Stereotactic radiosurgery for lung tumors: preliminary report of a phase I trial. Ann Thorac Surg. 2003;75:1097-101.

6. Pennathur A, Luketich J, Heron D, Abbas G, Burton S, Chen M, et al. Stereotactic radiosurgery for treatment of stage I non small cell lung cancers in high risk patients. J Thorac Cardiovasc Surg. 2009;137:597-604.

7. Harley DP, Krimsky WS, Sarkar S, Highfield D, Aygun C, Gurses B. Fiducial marker placement using endobronchial ultrasound and navigational bronchoscopy for stereotactic radiosurgery: an alternative strategy. Ann Thorac Surg. 2010;89:368-74.

8. Herth FJ, Becker HD, Ernst A. Ultrasound-guided transbronchial needle aspiration: an experience in 242 patients. Chest. 2003;123:604-7.

9. Beckles MA, Spiro SG, Colice GL, Rudd RM, American College of Chest Physicians. The physiologic evaluation of patients with lung cancer being considered for resectional surgery. Chest. 2003;123(1 Suppl): 105S-14S. 
10. Yasufuku K, Nakajima T, Motoori K, Sekine Y, Shibuya K, Hiroshima K, Fujisawa T. Comparison of endobronchial ultrasound, positron emission tomography, and CT for lymph node staging of lung cancer. Chest. 2006;130: 710-8.

11. Herth FJ, Ernst A, Eberhardt R, Vilmann P, Dienemann H, Krasnik M, et al. Endobronchial ultrasound-guided transbronchial needle aspiration of lymph nodes in the radiologically normal mediastinum. Eur Respir J. 2006;28:910-4.

12. Al-Sarraf N, Aziz R, Gately K, Lucey J, Wilson L, McGovern E, et al. Pattern and predictors of occult mediastinal lymph node involvement in non-small cell lung cancer patients with negative mediastinal uptake on positron emission tomography. Eur J Cardiothorac Surg. 2008;33:104-9.

13. Hammoud ZT, Anderson RC, Meyers BF, Guthrie TJ, Roper CL, Cooper JD, Patterson GA. The current role of mediastinoscopy in the evaluation of thoracic disease. J Thorac Cardiovasc Surg. 1999;118:894-9.

14. Pretreatment evaluation of non-small-cell lung cancer. Am J Respir Crit Care Med. 1997;156:320-32.

15. Gould MK, Kuschner WG, Rydzak CE, Maclean CC, Demas AN, Shigemitsu H, et al. Test performance of positron emission tomography and computed tomography for mediastinal staging in patients with non-small-cell lung cancer: a metaanalysis. Ann Intern Med. 2003;139:879-92.

16. Herth FJ, Eberhardt R, Krasnik M, Ernst A. Endobronchial ultrasound-guided transbronchial needle aspiration of lymph nodes in the radiologically and positron emission tomography-normal mediastinum in patients with lung cancer. Chest. 2008;133:887-91.

\section{Discussion}

Dr Richard I. Whyte (Stanford, Calif). Dev, congratulations on a nice presentation, and thank you for sending me the manuscript in advance.

I have a couple of comments, and the questions I will put at the end.

I think this study nicely complements the paper we heard this morning about EBUS versus mediastinoscopy. Here, you used these patients who were undergoing bronchoscopic FM placement as your platform for this technique, and I think it should be noted that the use of FMs is clearly decreasing. As the technology of SBRT is getting better and better, the need for FMs is decreasing, and perhaps that will affect whether you do this, but I suspect not.

The focus of this paper, obviously, is on PET and CT. In this study, you used $5 \mathrm{~mm}$ as an indication for biopsy, and I was not really clear from the manuscript what you used as your criteria for PET and CT, whether it was $10 \mathrm{~mm}$ or $5 \mathrm{~mm}$ in the short-axis diameter or what the standard uptake value was. Interestingly, you had flow cytometry as a criteria for being negative, and it would be interesting to know whether that plays a significant role. But I think, overall, you do indicate about a $15 \%$ false negative rate for mediastinal node disease, and that is in keeping with the published data. On a couple of your slides, however, I noticed that you called the PET and CT findings false positives, and I think on 1 slide, you said that 9 of 11 had a false-positive rate on CT and 4 of 9 on PET. I think this assumes that your EBUS is actually truly negative, and we do not really know that because we do not have pathologic confirmation, and I suppose these false-positive PET and CT findings could, in fact, be false-negative EBUS studies. In general, I think that your conclusion that EBUS does upstage and downstage some tumors in some patients is absolutely correct. I think as to whether it is absolutely necessary, particularly in this cohort of fairly sick patients, and whether that affects survival is somewhat unclear. We do not have survival data, and often their survival is more limited by their comorbidities.
Essentially my questions are: What was the effect of flow cytometry here? Was that really necessary, and did it affect which findings were truly called negative? What were your criteria for calling them abnormal on CT? Was it $5 \mathrm{~mm}$, which I think is somewhat unconventional, or was it really $10 \mathrm{~mm}$, which I think is a more conventional number. Would you agree that some of these, quote, "false positive" CT scans might, in fact, be false-negative EBUS tests?

Overall, I enjoyed the paper. Thank you.

Dr Krimsky. Thank you very much.

The issue with respect to the CT scans, I think, to be clear, it was when the radiologist interpreting the CT scans called them enlarged, and it was typically using a short-axis diameter of $10 \mathrm{~mm}$ or $1 \mathrm{~cm}$. I do not think the flow cytometry helped very much, for what that is worth.

Dr Shrager. What was the third question, Richard?

Dr Whyte. Some of these, quote "false-positive" and "falsenegative" CT and PET scans, could they really be false-negative EBUS tests?

Dr Krimsky. Certainly. We have another study we are in the process of finishing the data collection for to study the mediastinal results and EBUS results at our particular institution, because data, much like politics, is all local, and at least from the data we have so far, the correlation rate is nearly perfect. Thus, I think the answer is yes and yes, if that helps.

Dr Mark J. Krasna (Towson, Md). I will just follow-up with that. That was a good presentation.

Bill, I guess I will put the question back to you as a comment. I think your group has done a great job, especially since the Society for Thoracic Surgery meeting, incorporating mediastinal nodal staging. Similar to Dr Whyte, we are using FM-less systems; thus, we do not need to perform bronchoscopy for that reason; however, as you have trained us, we are using EBUS and mediastinoscopy. Thus, my only question is, have you or your surgical colleague, Dr Harley, considered using mediastinoscopy for those 4 potential EBUS false-negative findings? Again, I think it is crucial, and we do that for all our patients. If we have an EBUS-negative finding, the patient undergoes mediastinoscopy; however, if it is positive, we will not have the patient undergo SRS because it might be inappropriate therapy. Has that been done yet?

Dr Krimsky. I think had we not done the additional interrogation and studied our mediastinal data comparing 1 test, at least at our institution, with the other, than I think yes, but, however, the data seem to be consistent. I think, again, it does bring up the idea of stage N1 disease, because, as best I can tell, if you are beaming something over here and you have a hilar node down here, it is 2 separate things altogether. I hope that answers the question.

Dr Hiran C. Fernando (Boston, Mass). Congratulations on the presentation. I think this is really important to be doing in patients who are going to be treated with nonoperative therapies.

In the abstract, you say that there is a $16 \%$ false-negative rate, but you are talking only about CT staging, yet you presented data on PET. Thus, if you combine the CT and PET information, what is your true false-negative rate, and how much of that false-negative rate is $\mathrm{N} 1$ disease and how much of that false-negative rate is $\mathrm{N} 2$ disease?

Dr Sarwate. With the PET scan, we had a false-negative rate of $10 \%$ and a false-negative rate of about $16 \%$ with the CT scan.

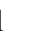


Three of 50 patients who underwent EBUS were positive for N1 disease on pathologic examination.

Dr Fernando. So, if you combine the CT and the PET information together, what is your true false-negative rate, using the best clinical staging that you would have up to this point?

Dr Sarwate. That would be around $8 \%$.

Dr Meyers. I am going to turn it around a little bit. Dr Fernando, you are the primary investigator for the very important American College of Surgeons Oncology Group/Radiation Therapy Oncology Group trial that is opening very soon, randomizing between SBRT, as discussed here, and sublobar resection. What sort of mediastinal evaluation are you requiring for that study that is opening soon?

Dr Fernando. Doing something like this would be perfect for the study, but I do not think we could really mandate that in all the sites, because 1 of the problems is patient accrual, and this study is going to be a 422-patient study, and to have all sites doing EBUS for every patient would not be practical. It would be perfect if we could do this.

Dr Meyers. So CT and PET?

Dr Fernando. We are doing CT and PET and then invasive mediastinal staging or EBUS or endoscopic ultrasonography for those lymph nodes that look suspicious by CT and/or PET.

Dr Paul Van Schil (Edegem, Belgium). Thank you for indeed for stressing a very important point, because radiation oncologists do not always pay enough attention to the lymph nodes.

I have 2 questions. You discovered N1 disease in some patients. Would you consider adding chemotherapy to the radiotherapy for those patients?

Dr Krimsky. Granted, there is a lot of controversy with that situation right now. I think we have left that to the discretion of the medical oncologists, but it is certainly an interesting consideration, especially in this patient population.

Dr Van Schil. Second, how far should you go with this staging? Current guidelines state that when you have negative EBUS findings and a suspicious lymph node on CT or PET, you should go for an invasive staging procedure. Would you consider mediastinoscopy in some patients to include the mediastinum in the radiation field in case you find N2 disease?

Dr Krimsky. I think that brings us back to Dr Krasna's question. We had been doing that, but we then looked at the data generated from mediastinoscopy, as well as the data generated from EBUS, and, given the correlation, it seemed to be reasonable to proceed at that point with just the EBUS findings alone. In addition, again, N1 disease is not picked up, so you are stuck with either thoracoscopy or something else if you are looking at the hilar.

Dr Joseph B. Shrager (Stanford, Calif). I would like to make a brief comment.

It is interesting that the radiation oncologists have moved in general before SBRT to using just PET to decide what their fields are going to be, and in a number of places, that is all they do. So we have to kind of bring them back and remind them that they should not be doing that with SBRT and that they are using an absolutely useless therapy if they have not evaluated the mediastinal nodes for patients who are at any risk of having mediastinal nodal disease.

Dr Meyers. You can say that, but their failure rate is not $16 \%$. It is much lower than that in patients they have selected. So, there might be some paradox going on with the kinder immune response after SBRT, but they do not fail at that high rate.

Dr Shrager. It is possible.

Dr Joachim Schirren (Wiesbaden, Germany). Excellent results.

You showed us that the staging in the mediastinum, with all the technical aspects, is never correct; therefore, the question: What will you do with the high-risk patient who receives SBRT? My question is, could you imagine that video-assisted mediastinoscopic lymphadenectomy or transcervical extended mediastinal lymphadenectomy could be the right treatment of this patient, that we have a very good resection of the lymph nodes without compromising the patient, and then this procedure will be better for this high-risk patient?

Dr Krimsky. In other words, surgery versus_-perhaps I did not understand the question.

Dr Schirren. I think the nodes, they are never cleared completely; therefore, perform video-assisted mediastinoscopic lymphadenectomy or transcervical extended mediastinal lymphadenectomy and resect the nodes. This is not compromising for the patient. You have the nodes resected. You have a very good staging, better staging, just as with EBUS. I like EBUS, but not in such cases, and then you can send this patient for SBRT.

Dr Krimsky. Perhaps, yes.

Dr Shrager. Are you familiar with video-assisted mediastinoscopic lymphadenectomy and transcervical extended mediastinal lymphadenectomy, these methods of minimally invasively resecting all the nodes?

Dr Krimsky. Yes.

Dr Kazuhiro Yasufuku (Toronto, Ontario, Canada). Thank you very much for the excellent presentation.

I have 1 comment and 1 question. I think the point that we can get N1 nodes is a big value of EBUS, because with SBRT, we can assess the mediastinum with mediastinoscopy, but we cannot get the N1 nodes with mediastinoscopy. From the results of your retrospective study, what would your recommendations be for performing EBUS for patients who are considered for SBRT? Would you do EBUS for all patients, regardless of the CT or PET findings, or would you just include patients who have positive CT or PET findings?

Dr Krimsky. I think that is a great question, but I think actually our results bear out what is in fact generally accepted in terms of the size/staging in terms of the lesion itself. We are finding a number of these are in lesions that are $2 \mathrm{~cm}$ or greater, but those $2 \mathrm{~cm}$ or less, we find a lot fewer. So, I think right now it seems that that guideline and that suggestion still seems to hold a lot of value. Again, it is another procedure. I think what Drs Whyte and Krasna also brought up is, you know, do you need FMs, and this just happened to be something that was concomitant for us. I hope that answers the question. 